\title{
Perceived coercion in the EUNOMIA study groups - a cross-national analysis of its intensity
}

\section{Lars Kjellin}

\author{
Address: Psychiatric Research Center, P.O. Box 1613, SE-70116 Örebro, Sweden \\ from WPA Thematic Conference. Coercive Treatment in Psychiatry: A Comprehensive Review \\ Dresden, Germany. 6-8 June 2007 \\ Published: 19 December 2007 \\ BMC Psychiatry 2007, 7(Suppl I):S99 doi:10.1 I86/I47I-244X-7-SI-S99
}

This abstract is available from: http://www.biomedcentral.com/I47I-244X/7/SI/S99

(C) 2007 Kjellin; licensee BioMed Central Ltd.

\section{Background}

To compare levels of perceived coercion at admission and during hospitalization among legally involuntarily and voluntarily admitted patients, and patients' perceptions of coercion at admission assessed by staff, across the EUNOMIA study sites.

\section{Methods}

More than 4,500 legally voluntarily admitted patients in 13 psychiatric services in twelve European countries were screened for perceived coercion at admission according to the MacArthur Perceived Coercion Scale (MPCS). Patients in this way identified as legally voluntarily admitted patients who felt coerced to admission, and consecutively legally involuntarily admitted patients, in all more than 3,400 patients, were interviewed and assessed at the end of the first week, at four weeks and at three months after admission. Perceived coercion was assessed by the MPCS and the Coercion Ladder (CL). Assessments on the CL were done by both patients and staff.

\section{Results}

The proportion of legally voluntarily admitted patients who felt coerced to admission ( $=3$ on the MPCS) ranged from 15 to $38 \%$ across the centers. For the legally involuntarily admitted patients, the proportion scoring high (=3) on the MPCS ranged from 77 to $98 \%$ and the proportion scoring high $(=5)$ on the CL from 53 to $98 \%$ across the centers. The staff assessed the amount of pressure from others, that they thought the patients experienced when they were admitted, to be lower compared to the patients' own reports. During stay in hospital, $17-95 \%$ of the involuntary patients, and between $16-72 \%$ of the voluntary patients who felt coerced to admission, scored high on the CL.

\section{Conclusion}

There was a considerable variation in levels of perceived coercion, at admission and during stay in hospital, across the study sites among legally voluntarily as well as legally involuntarily admitted patients. The patients' and the staff's assessments of perceived coercion differed. 